\title{
EXPLORING THE HELIOSPHERE IN THREE DIMENSIONS \\ A KEYNOTE PRESENTATION
}

\author{
L. A. FISK \\ Department of Atmospheric, Oceanic and Space Sciences, \\ University of Michigan, Ann Arbor, MI 48109 U.S.A.
}

\begin{abstract}
The 28th ESLAB Symposium marks the beginning of the sprint of the Ulysses mission to the very high heliographic latitudes and the pole-to-pole passage. The more than twenty-year quest to understand the Sun and the heliosphere in three dimensions is about to be realized. It is perhaps worthwhile, as we are poised to begin this journey, to ask how history is likely to judge this mission, or equivalently: what questions need to be answered so that the judgment will be kind?
\end{abstract}

\section{Introduction}

This Symposium marks the beginning of the sprint to high latitudes for the Ulysses spacecraft. We will assess where we are, having just completed the journey through the mid-heliographic latitudes, and position ourselves for the sprint to the very high heliographic latitudes, after which we will execute the pole-to-pole passage, and, hopefully, thereafter have a continued mission to study the heliosphere during solar maximum conditions.

It is, perhaps, worthwhile to remember how long it has taken us to get here. It was twenty years ago this year when a small group assembled in ESTEC to conceive the modern out-of-the-ecliptic mission. There had been considerable thought and encouragement given to out-of-the-ecliptic missions earlier, dating back even into the 1960's. However, the modern mission, the present-day Ulysses, had its origins in that ESTEC meeting.

Ian Axford (1994) has written a marvelous article for the 75th anniversary of the American Geophysical Union, reminiscing on his career, in which he discusses the events surrounding the conception and advocacy of the Out-of-the-Ecliptic mission, which became known as the International Solar Polar Mission, and now Ulysses. Those of us who attended that ESTEC meeting also have our recollections, with Ian standing at the blackboard, drawing the two-spacecraft concept, with one going over the north pole of the Sun and the other the south pole, and noting how they looked like two railroad trains racing in opposite directions at each other.

One of the trains, of course, was derailed along the way. However, the other has fulfilled our hopes over twenty years and longer, of finally being able to answer the simple, but compelling question: What does the heliosphere look like in three dimensions? In many ways it is such a basic question. The heliosphere is obviously a three-dimensional structure. We have every reason to expect that conditions vary markedly with latitude. We know that prior to Ulysses our sampling of the heliosphere near the equatorial plane of the Sun, although extensive, is non-representative of 
heliospheric conditions, and, moreover, that conditions at other latitudes, beyond our current observations, will influence what happens near the equatorial plane. It is, perhaps, a wonder that it has taken us so long to fill this obvious gap in our knowledge and understanding of what has been a central subject in space research, the Sun and the heliosphere.

Rather than dwell on this past, however, it is probably more worthwhile to consider Ulysses and the exploration of the three-dimensional heliosphere from the perspective of the future. As we begin the sprint to high latitudes and the pole-to-pole survey, we should ask what we expect to learn, or more appropriately, we should consider that we are reading the history of this mission twenty years from now, and ask what is likely to be said about our impact. These exercises are useful if for no other reason than to call our attention to the larger issues. We all tend to work on problems that most interest us, even if they are very focused and of a detail interesting only to a few. We will be judged, however, on whether we addressed the larger issues. Was this a seminal mission that laid to rest uncertainties that had plagued the field for many years? Did we make new discoveries that opened up whole new areas of research? Did we have an impact on fields beyond our own?

Consider a possible list of the larger issues, or equivalently, the several themes which unify the several investigations that Ulysses will pursue-and which, if successfully completed, history will judge this mission kindly. Essentially there are four: The first is to determine the overall morphology of the solar wind, its embedded magnetic field, and its accompanying waves and turbulence. The second is to determine the behavior of galactic cosmic rays. The third is to trace the behavior of the interstellar gas which enters the heliosphere as neutral particles and which departs as the anomalous cosmic ray component. The fourth is to understand the source of the solar wind and energetic particles in the solar corona and lower solar atmosphere.

The first theme-the overall morphology of the solar wind-is a matter of making in situ observations. The fourth theme-inferring coronal processes-is a matter of making remote observations. The second theme-understanding cosmic ray behavioris a matter of inferring where the particles have been in physical space. The third theme-the interstellar ions-is a matter of inferring where the particles have been in energy space. Consider each of these problems separately.

\section{The Overall Morphology of the Solar Wind}

This theme embodies the exploration aspect of Ulysses-go where we have never been before and provide a complete description of the heliosphere at all latitudes for at least this portion of the solar cycle. The observations should fall in three categories:

First, we will confirm what we expect to find, and, as is evident in the papers of this Symposium, have found to date with Ulysses. We have every expectation that the solar wind is faster over the solar poles originating in the large coronal hole present there. We expect that there will be fewer effects from stream-stream interactions. We 
expect that the polarity of the magnetic field will be uniform. We expect that the magnetic field, on average, will be more radial. Indeed, if there are marked deviations near the solar poles from these expectations, that will be significant.

The second category of observations is the one that will settle controversies. There is some expectation (Jokipii and Kota, 1989) that although the magnetic field is on average radial over the solar poles, its weakness, its presence in a strong radial outflow, and with strong rotational effects over the poles, there could be significant non-radial terms present. These deviations of the magnetic field from its average configuration, if they occur, could have a strong influence on galactic cosmic ray propagation, but it will remain for Ulysses to determine whether there is evidence that the fluctuations exist.

Finally, we have the category of observations that is characteristic of all exploratory missions-the observations that will be a surprise. We have a picture of MHD fluctuations in the solar wind, which results from our observations near the equatorial plane-the fluctuations appear to be more wave-like and of solar origin in the inner heliosphere and more characteristic of locally generated turbulence in the azimuthal magnetic fields of the outer heliosphere (see, e.g. Roberts and Goldstein, 1991). What will Ulysses observe admittedly at essentially one radial distance, in the radial field over the solar poles? We have an understanding near the equatorial plane of the influence of stream-stream interactions on the dynamics and internal structure of the solar wind. What will Ulysses see in the polar regions where stream-stream interactions are absent?

By simply going to high latitudes and documenting what is observed, this aspect of Ulysses is bound to be a success. The data obtained should make it possible to construct an overall model for the three-dimensional heliosphere during the current, solar minimum portion of the solar cycle.

\section{The Behavior of Galactic Cosmic Rays}

The second unifying theme-understanding at long last the behavior of galactic cosmic rays in the heliosphere-also has a reasonable chance of success. In many ways, this problem has the same twenty year history as does Ulysses. In the late 1960 's and early 1970's it was recognized that adiabatic deceleration of the cosmic rays in the expanding solar wind would be important (see, e.g. Parker, 1965; Gleeson and Axford, 1967). Particles, on average, would loose several hundred MeV/nucleon in energy, which is not a major effect for high energy particles but which effectively precludes particles with energies of less than 100-200 MeV/nucleon in the interstellar medium from penetrating to the inner heliosphere near the equatorial plane (see, e.g. Goldstein et al., 1970). In the mid-1970's it was recognized that latitude effects would be important. The intrinsic difference between low heliographic latitudes, where the magnetic field is primarily azimuthal, and the polar regions, where it is primarily radial, should give easier access over the solar poles. Thus, large latitude gradients should be introduced, 
which with cross-field transport, makes latitude effects significant at all latitudes (see, e.g. Fisk, 1976). Finally, in the late 1970 's and throughout the 1980's, when the overall organization of the magnetic structure of the heliosphere was understood, with one polarity in one hemisphere and the opposite polarity in the other, and with a current sheet between whose orientation varies with the solar cycle, the role of gradient and curvature drifts, the ultimate transport mechanism in latitude, was recognized (see, e.g. Jokipii, 1986).

Throughout all this evolution in understanding, the basic physics, or equivalently, the governing equation for cosmic ray behavior has not changed:

$$
-\nabla \cdot \mathbf{V}\left(\frac{p}{3}\right)\left(\frac{\partial F}{\partial p}\right)=\nabla \cdot(\mathbf{K} \cdot \nabla F)-\mathbf{V} \cdot \nabla F-\mathbf{v}_{\mathbf{D}} \cdot \nabla F
$$

This is the equation for the steady-state behavior of the cosmic ray distribution function, $F$, in terms of its momentum $p$. It is a balance between several effects: (i) spatial diffusion (the first term on the right), which is usually described by a phenomenologically determined diffusion tensor, $\mathrm{K}$, which governs the tendency of the cosmic rays to relax to isotropy in the frame of the solar wind; (ii) convection with the solar wind speed, $\mathbf{V}$ (the second term on the right); (iii) adiabatic deceleration in the expanding solar wind (the term on the left); and (iv) a term (the third on the right) which describes the drift of the particles with an effective drift speed, $\mathbf{v}_{\mathbf{b}}$, in the mean magnetic field of the heliosphere. An explicit time dependence can be added to this equation through a time derivative term and with, for example, a diffusion tensor that varies with time. Care also needs to be exerted in dealing with the drift term near the equatorial current sheet.

This equation is believed to be an accurate description of the cosmic ray behavior. The issue is not its likely accuracy, but rather what parameters to choose in applying the equation, or equivalently: How do the cosmic rays observed at a given location in the heliosphere reach this point? The equation is second order due to the diffusion term; however, for relatively high energy particles where the spatial gradients are not large, only the first order terms may be significant. The resulting approximate equation, then, describes the average trajectories of the cosmic rays, or the streamlines, in physical and momentum space, from the interstellar medium to where they are observed. In models where drifts in the mean magnetic field are important, and in which the magnetic field over the solar poles is essentially radial, the trajectory of the cosmic rays during the portion of the solar cycle seen by the upcoming polar pass of Ulysses should be inward over the solar poles and downward in latitude onto the equatorial plane (see, e.g. Jokipii and Kopriva, 1979). However, if the magnetic field over the poles is less radial and more disordered, with a resulting increase in diffusion and a reduction in the mean drift, then the average trajectory of the particles could be altered significantly (Jokipii and Kota, 1989). 
For several decades we have speculated on how cosmic rays behave in the solar wind and what causes their solar cycle variations. We have models with warped current sheets, which should be important when the magnetic cycle of the Sun is such that the drift of the cosmic rays is inward along the current sheet (see, e.g. Smith and Thomas, 1986). We have models in both halves of the magnetic cycle of the Sun in which large stream-stream interaction regions are important for cosmic ray behavior (see, e.g. Burlaga et al., 1987). The details and the differences of these various models can be resolved only when we know the flow patterns of the cosmic rays-where they have been and what they have experienced-and this, through observations of the solar wind and its magnetic field, and the response of the cosmic rays, is information that Ulysses in principle can provide.

There may be an extra bonus also over the solar poles. If the access is easy, then adiabatic deceleration, which results in 100-200 MeV/nucleon energy loss for particles seen near Earth, may be reduced. The inward trajectory of cosmic rays over the solar poles may be sufficiently fast so that there is little time to lose energy, and low energy interstellar cosmic rays, which are unobservable elsewhere in the heliosphere, may be present. Similarly, the absence of solar energetic particles over the solar poles, should that be the case, may reveal heretofore unobserved portions of the galactic cosmic ray spectrum.

\section{The Behavior of Interstellar Gas in the Heliosphere}

The third unifying theme is to trace the behavior of interstellar gas in the heliosphere. Tracing the behavior of galactic cosmic rays was a matter of filling in the gap in our knowledge of their spatial behavior. Tracing the behavior of the interstellar gas is a matter of finding out where these particles have been in energy space.

Ulysses will make direct measurements of neutral interstellar helium as it is swept into the heliosphere by the motion of the Sun relative to the local interstellar medium (Witte et al., 1992). However, there is much more to the behavior of the interstellar gas than just its neutral component. There is the behavior of pick-up ions-interstellar gas as it becomes ionized by photo-ionization from the Sun and charge-exchange with the solar wind (Gloeckler and Geiss, 1994) - and there is the anomalous component. This problem also spans a time period identical to the development of Ulysses. In 1973 and 1974, the anomalous component-cosmic rays with energies in the range 10$100 \mathrm{MeV} /$ nucleon with the anomalous composition of mainly helium, nitrogen and oxygen-was observed (Garcia-Munoz et al., 1973; Hovestadt et al., 1973; McDonald et al., 1974). Shortly afterwards the anomalous component was identified by Fisk $e t$ al. (1974) as likely to originate as interstellar neutral gas that is ionized and accelerated in the solar wind. It has remained, however, for two decades to find compelling evidence that this origin is correct. The principal prediction of Fisk et al. was that the particles would be singly-charged, a prediction now confirmed by observation (see, e.g. Adams et al., 1991). It remained for Ulysses en route to Jupiter to observe the recently ionized 
neutrals, principally the hydrogen and oxygen, shortly after they are picked up by the solar wind-the so-called pick-up ions. (Gloeckler and Geiss, 1994 ).

There is still, however, a major issue with this problem. Somewhere in the solar wind, but not of certain location, a very effective acceleration mechanism is occurring. When the interstellar ions are picked up by the solar wind, they acquire energies of approximately $1 \mathrm{keV} /$ nucleon. When they are observed as the anomalous component, they have energies of order tens of $\mathrm{MeV} /$ nucleon, or a four order of magnitude increase.

The prevailing explanation is that the interstellar particles are accelerated at the termination shock of the solar wind, as was suggested by Pesses et al. (1981). The acceleration has to occur in the outer heliosphere, since the intensity seen by Pioneer and Voyager increases with heliocentric distance (see, e.g. Cummings et al., 1994). Presumably, there is a shock present since the supersonic solar wind has to become subsonic to merge with the interstellar medium. It surrounds the heliosphere giving a good shock surface for acceleration, and shocks are known to be good accelerators. However, we should perhaps always be somewhat cautious about prevailing explanations for which there is no direct observational support and only circumstantial evidence. Indeed, there may be some potential concerns with the termination shock being the sole accelerator of the anomalous component.

In a recent paper, Cummings et al. (1994) estimated the pressure in the anomalous component at the termination shock by extrapolating Voyager data out to the likely shock location and by assuming that the shock is strong, i.e. a jump in the flow velocity by a factor of 4 . They found that the pressure was only about $2 \%$ of the ram pressure of the solar wind. Had they done their calculations for a weaker shock, e.g., a velocity jump by $2-3$, they would have found that the required pressure was dramatically higher, perhaps $40-50 \%$ of the ram pressure.

On the other hand, observations of Gloeckler $e t$ al. (1994) from Ulysses indicate that there may be considerable pressure in the pick-up particles, particularly if, as seems to be the case, there is additional acceleration of the pick-up ions in, e.g., Corotating Interaction Regions. An extrapolation of the observed pick-up pressure to the location of the termination shock yields a possible pressure in the pick-up ions of $10-15 \%$ of the ram pressure.

Herein lies the concern about the termination shock as the sole accelerator of the anomalous component. If the pick-up ions are mobile in the solar wind and do not contribute significantly to the solar wind internal pressure, as is the case for galactic cosmic rays, they will not affect the solar wind Mach number. The termination shock will then be a strong shock, with a velocity jump of order 4 . However, if the pick-up ions are mobile in the solar wind, they should also be readily injected into the shock acceleration mechanism. The resulting pressure then in the accelerated anomalous particles will exceed the $10-15 \%$ of the ram pressure in the pick-up ions, and be much larger than the $2 \%$ inferred by Cummings et al. (1994).

Conversely, if the pick-up ions are not able to move easily in the solar wind, as is probably the case, they will determine the solar wind Mach number. At a pressure of $10-15 \%$ of the ram pressure, they render the termination shock a relatively weak shock 
and their heating at the shock is responsible for pressure balance. For example, if there is considerable pressure in the anomalous component, there should be a strong fore shock region, which slows the upstream solar wind and adiabatically compresses the embedded pick-up ions. It can readily be shown that the pressure allowed in the anomalous component, so that the adiabatically compressed pick-up ions, the anomalous component, and the solar wind ram pressure are all in pressure equilibrium, is much less than the $40-50 \%$ required to explain the observations of Cummings et al. (1994) in the weak shock case.

These conclusions depend on the pressure in the pick-up ions being significant at the termination shock (10-15\% of the ram pressure). Should this be the case, we should be concerned about the role the termination shock plays in the acceleration of the anomalous component.

The role that observations from Ulysses will have in determining the acceleration mechanism for the anomalous component will depend in part on what the mechanism is. If the acceleration occurs at the termination shock, Ulysses has the opportunity to probe conditions at the shock at different latitudes. The shock strength could vary with latitude, as well as the ability of particles to be injected into the shock acceleration mechanism since the direction of the magnetic field relative to the shock normal will vary with latitude. These differences may be revealed in the behavior of the anomalous component with latitude. Conversely, if the termination shock is not the only accelerator, and this is determined either by direct observations from Pioneer and Voyager or by inference because the magnitude of the pressure in pick-up ions and its role in the strength of the termination shock is understood, then the latitude observations of Ulysses could have the important role of determining what the principal acceleration mechanism actually is.

\section{Coronal Processes}

The fourth and final unifying theme is to use observations from Ulysses to access remotely coronal processes; in particular, the heating and acceleration of the solar wind and the acceleration and propagation of energetic particles.

The basic concepts of how the solar wind is accelerated have, of course, been known since the late 1950's (Parker 1958). However, "the devil is in the details", some of which are quite fundamental: How is the corona heated? How are high speed streams with the correct speed, density, and temperature generated? How are such basic properties as electron heat conduction included correctly, or what is the role of open and closed magnetic field lines in the transition region and lower corona? How do we model this dynamic, time-varying process, or, equivalently, how important are timevarying effects for the models which are essentially steady-state?

Ulysses, of course, gives us three advantages in being able to contribute to understanding the acceleration of the solar wind. The first is perspective. Ulysses gets to look down the throat of the solar wind by observing the solar wind as it comes 
directly from a large polar coronal hole, relatively undisturbed by non-radial flow through the corona or by interactions between high and low speed streams. Second, Ulysses carries very capable plasma instruments, including one that can measure the elemental and charge composition of the solar wind (Gloeckler et al., 1992). Since the charge states of the solar wind are frozen-in in the corona and the elemental composition is altered by coronal processes and in the lower solar atmosphere, these observations can place serious constraints on acceptable models for coronal heating and solar wind acceleration. Finally, there should be an opportunity for detailed coordinated measurements between Ulysses measuring the product of the solar wind acceleration and Spartan 201 measuring the coronal conditions that give rise to this wind. It will be a rare opportunity to look at the solar wind acceleration process from both ends.

Studies of solar energetic particles will also be significant. The issue here should be primarily coronal propagation. The sources of solar cosmic rays are primarily at low and mid-latitudes. By observing at high latitudes, particularly over a large coronal hole, Ulysses should provide a unique perspective on the mechanisms by which energetic particles propagate in the corona.

\section{Conclusions}

Determine the overall morphology of the heliosphere-the large-scale variations of the solar wind and its magnetic field; the behavior of its turbulence. Trace where the galactic cosmic rays have been. Reveal the influence of interstellar ions and determine where and how the acceleration of the anomalous component is occurring. Contribute to our understanding of the acceleration of the solar wind and the coronal propagation of solar energetic particles. It is a pretty good list. Certainly not a complete one, for there are other significant investigations on Ulysses, e.g., the measuring of the properties of dust in the heliosphere and the studying of solar x-ray emission. However, a good list nonetheless, which if executed successfully will allow history to judge kindly the Ulysses mission and our twenty-year odyssey to explore the heliosphere in three dimensions.

\section{References}

Adams Jr., J. H. et al. : 1991, Astrophys. J. (Letters) 375, L45.

Axford, W. I.: 1994, J. Geophys. Res., in press.

Burlaga, L. F., Ness, N. F., and McDonald, F. B.: 1987, J. Geophys. Res. 92, 13647.

Cummings, A. C., Stone, E. C., and Webber, W. R.: 1994, J. Geophys. Res., in press.

Fisk, L. A.: 1976, J. Geophys. Res. 81, 4646.

Fisk, L. A., Kozlovsky, B., and Ramaty, R.: 1974, Astrophys. J. (Letters) 190 L35.

Garcia-Munoz, M., Mason, G. M., and Simpson, J. A.: 1973, Astrophys. J. (Letters) 182, L81.

Gleeson, L. J. and Axford, W. I.: 1967, Astrophys. J. (Letters) 149, L115.

Gloeckler, G. and Geiss, J.: 1994, Cosmic Winds and the Heliosphere, ed. by J. R. Jokipii and C. Sonett, University of Arizona Press, Tucson, in press.

Gloeckler, G. et al. : 1992, Astron. and Astrophys (Suppl) 92, 267.

Gloeckler, G. et al. : 1994, J. Geophys. Res., in press. 
Goldstein, M. L., Fisk, L. A. and Ramaty, R.: 1970, Phys. Rev. Letters 25, 832.

Hovestadt, D. et al. : 1973, Phys. Rev. Letters 31, 650.

Jokipii, J. R.: 1986, The Sun and the Heliosphere in Three Dimensions, Reidel Pub., Dordrecht, pp 375-388.

Jokipii, J. R. and Kopriva, D. A.: 1979, Astrophys. J. 234, 384.

Jokipii, J. R. and Kota, J.: 1989, J. Geophys. Res. Letters 16,1.

McDonald, F. B. et al. : 1974, Astrophys. J. (Letters) 249, L71.

Parker, E. N.: 1958, Astrophys. J. 128, 664.

Parker, E. N.: 1965, Planet. Space Sci. 13, 9.

Pesses, M. E., Jokipii, J. R., and Eichler, D.: 1981, Astrophys. J. (Letters) 246, L85.

Roberts, D. A. and Goldstein, M. L.: 1991, U. S. Nat. Report. to Inter. Union of Geodesy and Geophysics 1987-1990, pp 932-943.

Smith, E. J. and Thomas, B. T.: 1986, J. Geophys. Res. 91, 2933.

Witte, M. et al.: 1992, Astron and Astrophys (Suppl.) 92, 333. 